\title{
Avaliação dos resultados tardios de mamoplastia redutora pela técnica periareolar
}

\section{Late results after mammaplasty by the periareolar approach}

José Horácio ABOudiB ${ }^{1}$ Ana Claudia Weck Roxo²

Trabalho realizado no Hospital Universitário Pedro Ernesto Universidade Estadual do Rio de Janeiro, Rio de Janeiro, RJ, Brasil.

Artigo submetido pelo SGP (Sistema de Gestão de Publicações) da RBCP.

Artigo recebido: 8/7/2009 Artigo aceito: $17 / 3 / 2011$

\begin{abstract}
RESUMO
Introdução: Foram selecionadas aleatoriamente 25 pacientes submetidas a mamaplastia redutora pela técnica periareolar, com no mínimo 10 anos de pós-operatório. Em nenhum momento houve o espírito de avaliar comparativamente com outros tipos de técnica em uso no arsenal da cirurgia de redução e pexia mamária. Objetivo: Avaliar os benefícios e as desvantagens desta técnica a longo prazo. Método: Foram selecionados e analisados critérios como tamanho, formato, simetria, sensibilidade no complexo aréolo-papilar, qualidade das cicatrizes, assim como a opinião das pacientes, além do registro de complicações, reintervenções e do grau de satisfação. Todas as pacientes responderam a questionários específicos, permitindo conclusões avaliadas percentualmente para cada tipo de variável. Resultados: Dentre os comentários obtidos, a maioria das pacientes concordou que se submeteria a nova mamaplastia pela técnica periareolar. $\mathrm{O}$ volume e a forma das mamas mantiveram-se estáveis e com elevado grau de satisfação por parte das pacientes. A queixa mais frequente foi relacionada à cicatriz. Conclusão: $O$ resultado da análise comprovou que a abordagem periareolar é um procedimento muito útil para as mamaplastias redutoras em pacientes com pequena a média hipertrofia, sem ptose marcante e com aceitável a boa elasticidade cutânea. A principal desvantagem, entretanto, é a tendência da cicatriz ao redor da aréola alargar com o tempo.
\end{abstract}

Descritores: Mamoplastia/métodos. Mama/cirurgia. Procedimentos Cirúrgicos Reconstrutivos. Cirurgia Plástica.

\begin{abstract}
Introduction: Twenty five female patients submitted to breast reduction by periareolar approach for at least 10 years follow-up were selected. There was no intent to compare the technique with others routinely used for mammaplasty or only mastopexy. Objective: To evaluate the benefits and disadvantages of this technique in a long term follow-up. Methods: Several criteria were analyzed such as volume, shape, symmetry, nipple-areola complex sensibility, scars quality, as well as patient's opinion of their aesthetic results. Besides that, complications and re-intervention rates and satisfaction grade were also considered. Results: The majority of the patients accepted to be submitted to mammaplasty by periareolar approach. The breasts shape and volume remained stable with a high acceptable and satisfaction rates by the patients. The main complain was related to the scar's quality. Conclusion: The final analysis proved that the periareolar approach is a very efficient procedure in selected group of patient with small to mild breast volume, with small to moderate ptosis and with moderate to good skin turgor. The main disadvantage of the technique is the tendency of the scar to enlarge in long term post-operative period.
\end{abstract}

Keywords: Mammaplasty/methods. Breast/surgery. Reconstructive Surgical Procedures. Surgery, Plastic.

1. Professor de Cirurgia Plástica da Universidade Estadual do Rio de Janeiro; Chefe do Setor de Cirurgia Plástica do Hospital Universitário Pedro Ernesto - Universidade Estadual do Rio de Janeiro, Rio de Janeiro, RJ, Brasil.

2. Cirurgiã Plástica do Hospital Universitário Pedro Ernesto - Universidade Estadual do Rio de Janeiro, Rio de Janeiro, RJ, Brasil. 


\section{INTRODUÇÃO}

A satisfação das pacientes submetidas à cirurgia plástica estética em geral, e nas mamaplastias em particular, está sempre vinculada à qualidade dos resultados a curto, médio e longo prazo, com o mínimo de cicatrizes, à manutenção da sensibilidade cutânea e funcional, qualquer que seja o tipo de técnica aplicada. Apesar de seguir estes critérios, a técnica de redução e pexia mamária por via periareolar ${ }^{1-4}$ compreende um procedimento de indicações restritas, que oferece qualidade e satisfação às pacientes dentro destas características.

Bustos $^{5}$, em 1992, descreveu a técnica periareolar mediante o implante de uma tela de nylon, para melhor modelagem das mamas e maior efeito estético a longo prazo, com bons resultados, porém com índices elevados de complicações. Góes ${ }^{6-8}$, também pela via periareolar, tem utilizado tela de polipropileno, com menor incidência de reações tardias e com resultados a longo prazo satisfatórios. Benito \& Sanza ${ }^{9}$ não empregam nenhum tipo de tela ao redor do tecido mamário, porém realizam uma sutura em torno da aréola, tipo "round block", para minimizar o alargamento da cicatriz.

$\mathrm{O}$ procedimento que temos utilizado há mais de 15 $\operatorname{anos}^{10}$ segue os princípios de Bustos, porém não inclui o uso de tela e agrega alguns procedimentos cirúrgicos. Muitas alterações táticas e técnicas foram introduzidas ao longo dos anos ${ }^{11,12}$.

O objetivo deste trabalho é registrar os procedimentos que temos aplicado nas mamaplastias periareolares, avaliações sobre a qualidade dos resultados, as intercorrências e as complicações, além do estudo do grau de satisfação das pacientes com pelo menos 10 anos de pós-operatório.

\section{MÉTODO}

Numa série de 100 pacientes por nós operadas, 25 foram selecionadas aleatoriamente. Todas tinham no mínimo 10 anos de seguimento pós-operatório e eram portadoras de hipertrofia mamária moderada, ausência ou presença de ptose de grau I e bom turgor de pele.

A idade das pacientes variou de 16 a 49 anos, na época da cirurgia.

Foi coletado o maior número possível de informações que pudessem oferecer subsídios para validação da aplicação da técnica nas mamaplastias estéticas.

As pacientes receberam questionários com tópicos referentes a qualidade do resultado, volume final, forma e ptose das mamas, qualidade estética do complexo aréolo-papilar (CAP), simetria, qualidade da cicatriz e grau de satisfação.
Foram, ainda, coletados dados sobre complicações e reoperações. Além disso, as pacientes opinaram se fariam ou não novamente a cirurgia com o mesmo tipo de técnica. A cada um desses itens foram atribuídos notas e critérios que pudessem ser avaliados e respondidos por elas.

Apesar de todos os questionários terem sido elaborados pelos autores e analisados por um estatístico, as entrevistas com as pacientes foram realizadas por um profissional de saúde que não fazia parte da equipe cirúrgica, com o intuito de evitar constrangimento nas pacientes.

\section{Demarcações das mamas}

As demarcações das mamas são realizadas com a paciente sentada, o tronco ereto e sem medicação pré-anestésica. O ponto "A" é marcado como limite superior da aréola, cerca de $8 \mathrm{~cm}$ do sulco inframamário e não como o ponto (A') projeção do mamilo aplicado na técnica de Pitanguy ${ }^{13}$, e portanto, $2 \mathrm{~cm}$ acima do mesmo. O ponto "B" é demarcado de 4 a $6 \mathrm{~cm}$ acima do sulco inframamário, ambos correspondendo aos limites superior e inferior da aréola. As demarcações medial "C" e a lateral " $D$ " são posicionadas de acordo com o pinçamento bidigital da pele. Representam, respectivamente, os limites medial e lateral de desepitelização até a borda da aréola. O ponto externo " $D$ " deve ser mais próximo da borda da aréola do que o ponto "C", para evitar a lateralização da aréola (Figura 1). Há alguma subjetividade nesta marcação, o que exige certa experiência do cirurgião.

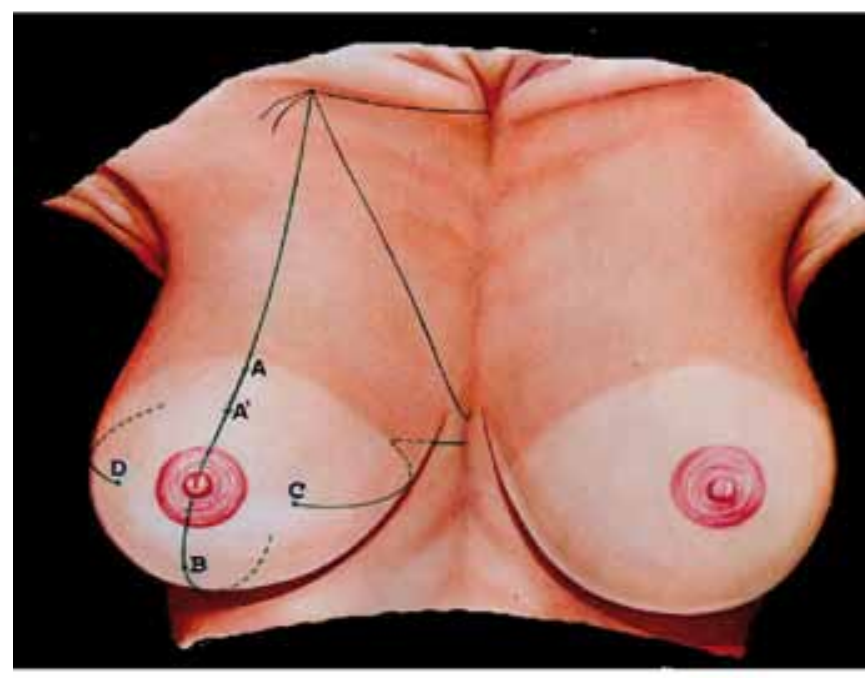

Figura 1 - Aspecto esquemático das demarcações cutâneas. Os pontos A e $B$ representam os limites superior e inferior da aréola a ser reposicionada. Distam, respectivamente, $8 \mathrm{~cm}$ e $4 \mathrm{~cm}$ do sulco inframamário. O ponto A'utilizado na técnica de Pitanguy representa a projeção do mamilo, porém não é utilizado como parâmetro. Os pontos $C$ e D representam os limites medial e lateral da aréola, sendo o ponto D alguns milimetros mais próximos dela do que $C$, para evitar a sua lateralização. 


\section{Técnica operatória}

As cirurgias são realizadas com as pacientes sob anestesia geral e infiltração local com epinefrina a 1:200.000 em todos os quadrantes a serem dissecados e areolótomo de $4.0 \mathrm{~cm}$ de diâmetro para determinar o novo limite circular externo das aréolas.

É realizada desepitelização na área delimitada pelas letras A, $\mathrm{B}, \mathrm{C}$, e D até o contorno da aréola demarcada pelo areolótomo (Figura 2). Procede-se à dissecção e à mobilização do parênquima mamário nos quadrantes superiores no sentido cranial, até atingir a aponeurose do músculo peitoral, em seguida caudalmente, até o nível do CAP (Figuras 3 e 4). Exteriorização dos quadrantes superiores, com a ressecção no sentido transversal de todo excesso existente acima do CAP, ao longo da linha demarcada pelo compasso (Figura 5). Nos casos de ressecção insuficiente, nova ressecção desses quadrantes em forma de cunha, no plano sagital, complementa o processo de redução volumétrica (Figura 6). Os dois quadrantes superiores (lateral e medial) são suturados entre si com maior giro do quadrante externo, com pontos isolados de nylon 3.0 e na aponeurose do músculo peitoral, recompondo o pólo superior da mama.

O limite da dissecção e o local de fixação dos pontos comporão o pólo superior do parênquima mamário (Figura

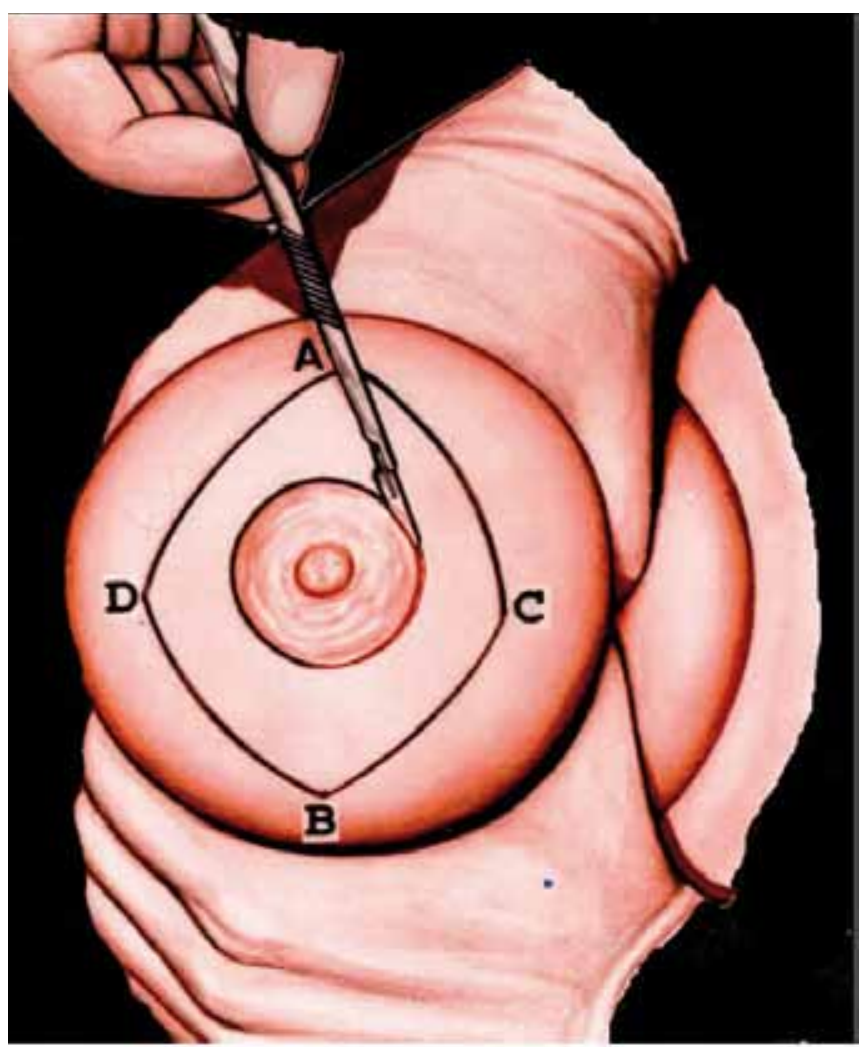

Figura 2 - Aspecto esquemático dos limites de pele a ser desepitelizada entre os quatros pontos $A B C D$ e a borda da aréola.

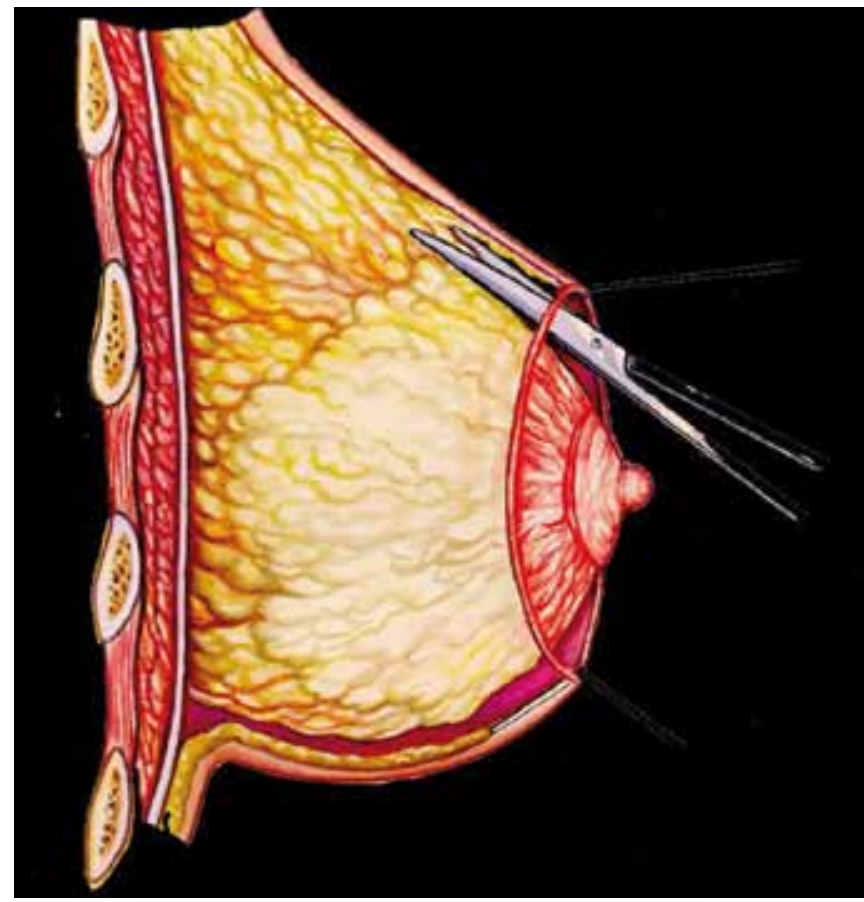

Figura 3 - Corte esquemático sagital da mama em que os quadrantes superiores são dissecados com tesoura nos limites do tecido glândulo-adiposo, até atingir a aponeurose do músculo peitoral maior.

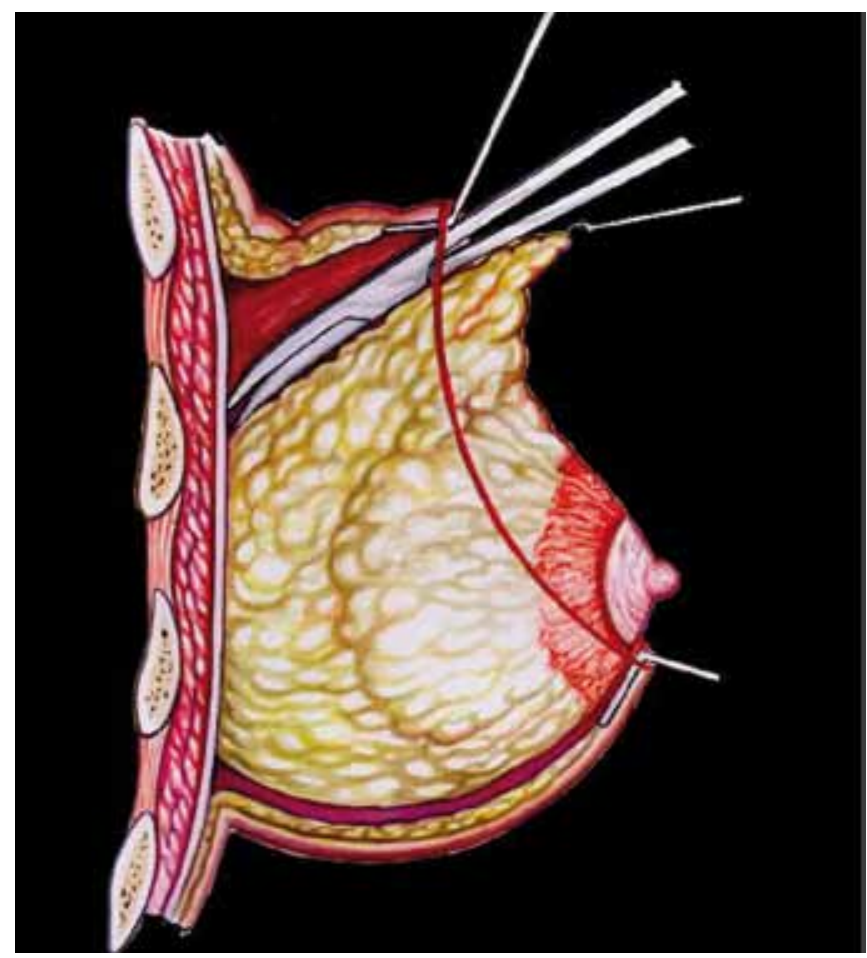

Figura 4 - Na continuação, a dissecção com tesoura se estende no mesmo plano da aponeurose do músculo peitoral no sentido caudal até o nível do plano horizontal da borda superior da aréola. 


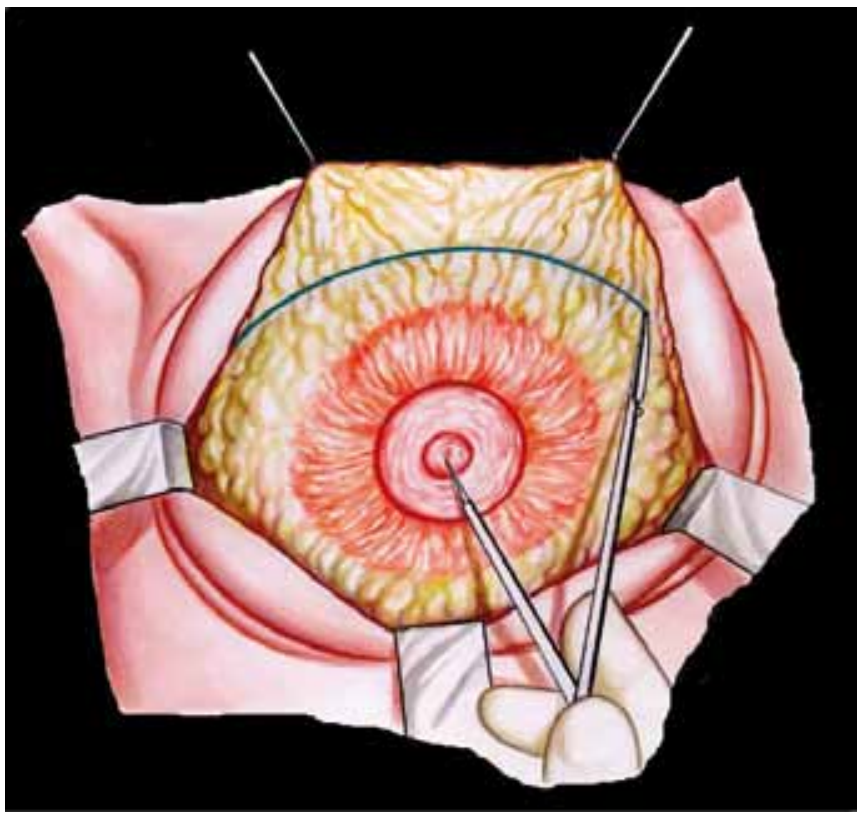

Figura 5 - Vista anterior da mama dissecada, em que a ressecção é delimitada mediante avaliação do cirurgião. Uma extremidade do compasso é aplicada no CAP e o outra nos limites da mama para a ressecção em bloco dos excessos nos quadrantes superiores.

A ressecção é realizada segundo uma cunha, compreendendo toda a espessura do tecido glândulo-adiposo no sentido da linha demarcada. Segue a sutura dos quadrantes superiores restantes ao plano músculo-aponeurótico do peitoral maior.

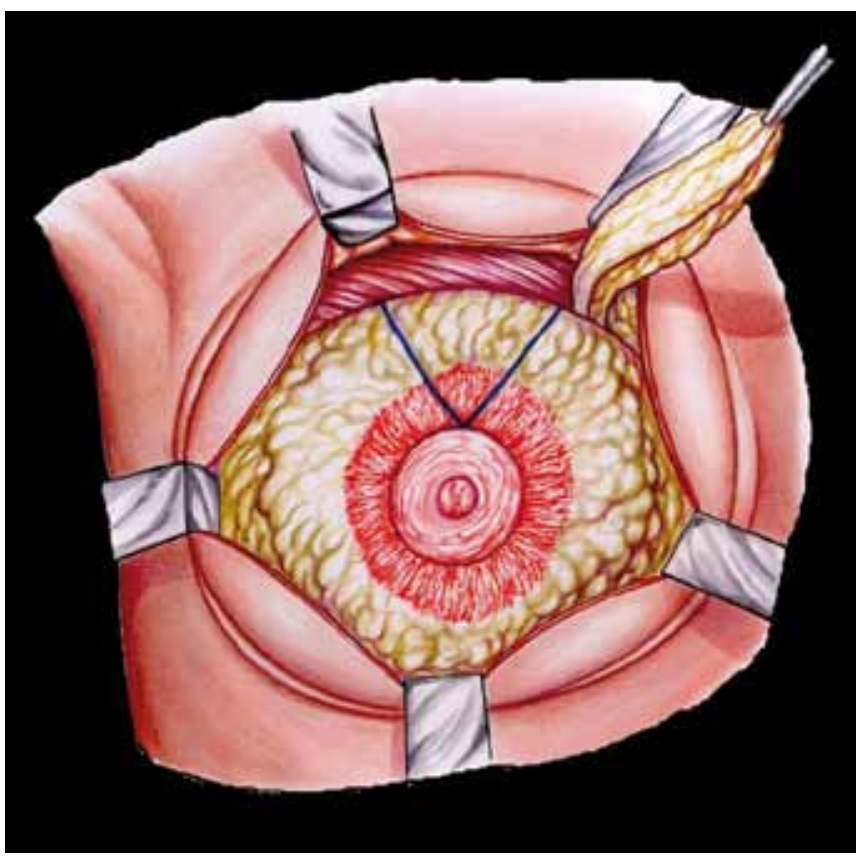

Figura 6 - Quando a ressecção dos dois quadrantes superiores é insuficiente, nova ressecção em cunha no plano sagital reduz ainda mais o volume.
7). Realiza-se a sutura contínua em “round-block”, no plano subdérmico, com nylon 3.0, de preferência com agulha reta (Figura 8). Fita adesiva porosa é aplicada diretamente sobre toda a aréola suturada. Drenos de aspiração a vácuo são aplicados pelo período de 24 a 48 horas. As mamas são recobertas com gaze e algodão. O tempo de internação é de 24 horas, passando a paciente a utilizar um sutiã cirúrgico antes da alta.

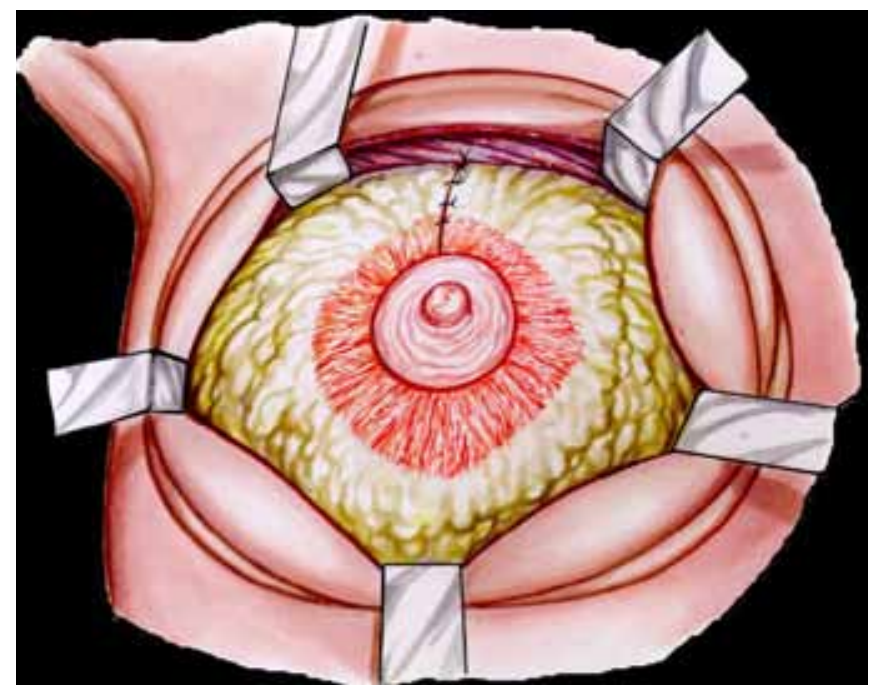

Figura 7 - Sutura realizada no plano sagital para o giro e conificação da mama.

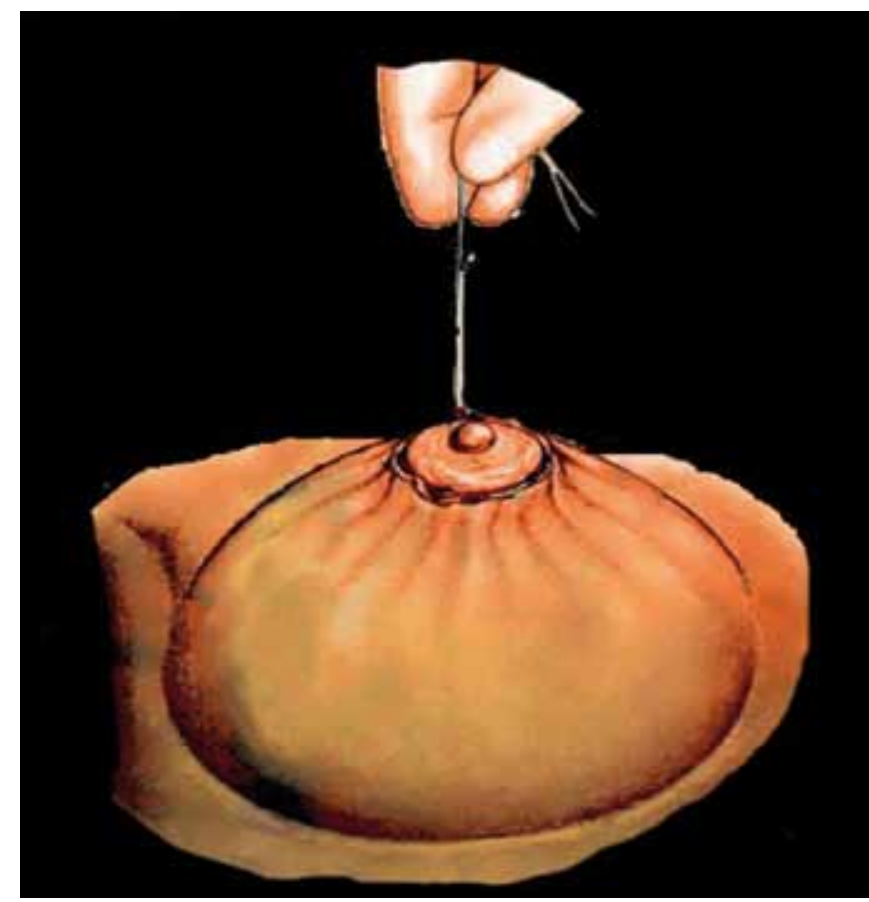

Figura 8 - Sutura final em "round-block “ da pele à borda da aréola. 


\section{RESULTADOS}

As pesquisas foram direcionadas para registrar o maior número de informações referentes às pacientes submetidas à mastoplastia periareolar. Logramos obter e tirar conclusões por meio de questionamentos após estudos estatísticos e sistematizados.

Quanto à idade das pacientes na época da cirurgia, $48 \%$ tinham menos de 19 anos, $52 \%$ entre 20 e 39 anos e $12 \%$ até 49 anos (Tabela 1 ).

A Tabela 2 demonstra o grau de satisfação das pacientes com o procedimento, avaliado com a aplicação de três notas: dois, um e zero, referentes a cinco aspectos: volume ressecado; forma e grau de ptose; qualidade do CAP; simetria das mamas; qualidade da cicatriz. Exceto para a qualidade do CAP com nota um, todos os demais itens receberam nota dois.

Quanto ao grau de satisfação com a diminuição do volume das mamas, nas reduções até $300 \mathrm{~g}$ em cada mama, $60 \%$ das notas foram entre 6 e 10 , nas reduções de 301 a $500 \mathrm{~g}, 24 \%$ tiveram notas também de 6 a 10 , e $16 \%$ receberam notas inferiores a 6 para qualquer volume de mama (Tabela 3).

A Tabela 4 registra $20 \%$ de complicações, dentre as quais $2(8 \%)$ casos de redução da sensibilidade no CAP unilateralmente, $2(8 \%)$ de ressecção insuficiente e 1 (4\%) de hematoma.

Quanto às reoperações, $10(40 \%)$ pacientes foram submetidas a revisão de cicatriz periareolar, uma (4\%) foi reoperada pela mesma técnica, e a outra (4\%) pela técnica de Pitanguy (Tabela 5).

Em relação ao grau de satisfação das pacientes com os resultados obtidos com o procedimento, $60 \%$ afirmaram estar muito satisfeitas, $28 \%$, satisfeitas, e $12 \%$, insatisfeitas (Tabela 6).

Quando questionadas quanto à técnica que escolheriam caso a cirurgia fosse realizada no momento da entrevista, $72 \%$ das pacientes responderam que repetiriam a mesma técnica, $20 \%$ optariam por outra técnica e $8 \%$ não se submeteriam a nenhum procedimento (Tabela 7).

A Tabela 8 especifica a somatória das respostas quanto à receptividade dos resultados obtidos em que foram dadas notas de zero a dez.

Tabela 1 - Idade das pacientes na época da cirurgia.

\begin{tabular}{c|c|c}
\hline Faixa etária & Número de pacientes & Porcentagem \\
\hline Abaixo de 19 anos & 12 & $48 \%$ \\
\hline 20 a 29 anos & 6 & $24 \%$ \\
\hline 30 a 39 anos & 4 & $16 \%$ \\
\hline 40 a 49 anos & 3 & $12 \%$ \\
\hline
\end{tabular}

Tabela 2 - Grau de satisfação das pacientes com o procedimento, quanto a volume ressecado; forma e grau de ptose; qualidade do CAP; simetria das mamas; qualidade da cicatriz.

\begin{tabular}{c|c|c|c|c|c}
\hline $\begin{array}{c}\text { Notas Grau de } \\
\text { satisfação }\end{array}$ & $\begin{array}{c}\text { Volume } \\
\text { ressecado }\end{array}$ & $\begin{array}{c}\text { Forma e grau de } \\
\text { ptose }\end{array}$ & $\begin{array}{c}\text { Qualidade do } \\
\text { CAP }\end{array}$ & $\begin{array}{c}\text { Simetria das } \\
\text { mamas }\end{array}$ & $\begin{array}{c}\text { Qualidade da } \\
\text { cicatriz }\end{array}$ \\
\hline Nota 2 & $22(88 \%)$ & $15(60 \%)$ & $19(76 \%)$ & $11(44 \%)$ \\
\hline Nota 1 & $2(8 \%)$ & $6(24 \%)$ & $11(44 \%)$ & $5(20 \%)$ & $11(44 \%)$ \\
\hline Nota 0 & $1(4 \%)$ & $7(16 \%)$ & $5(20 \%)$ & $1(4 \%)$ & $3(12 \%)$ \\
\hline
\end{tabular}


Tabela 3 - Idade das pacientes na época da cirurgia.

\begin{tabular}{c|c|c|c|c|c|c|c|c|c|c}
\hline Notas & $\mathbf{1}$ & $\mathbf{2}$ & $\mathbf{3}$ & $\mathbf{4}$ & $\mathbf{5}$ & $\mathbf{6}$ & $\mathbf{7}$ & $\mathbf{8}$ & $\mathbf{9}$ & $\mathbf{1 0}$ \\
\hline Menos $100 \mathrm{~g}$ & & & & & & & & & \\
\hline $101-200 \mathrm{~g}$ & & & & & & & & & \\
\hline $201-300 \mathrm{~g}$ & & & & & & & & & \\
\hline $301-400 \mathrm{~g}$ & & & & & & & & & \\
\hline $401-500 \mathrm{~g}$ & & & & & & & & & \\
\hline
\end{tabular}

Tabela 4 - Índice de complicações.

\begin{tabular}{c|c|c}
\hline Complicações & Número de Pacientes & $\%$ \\
\hline Nenhuma & 20 & $80 \%$ \\
\hline $\begin{array}{c}\text { Diminuição da sensibilidade } \\
\text { no CAP }\end{array}$ & 2 & $8 \%$ \\
\hline Hematoma & 1 & $4 \%$ \\
\hline Ressecção Insuficiente & 2 & $8 \%$ \\
\hline
\end{tabular}

Tabela 6 - Grau de satisfação.

\begin{tabular}{c|c|c}
\hline Complicações & Número de Pacientes & $\mathbf{\%}$ \\
\hline Muito Satisfeita & 15 & $60 \%$ \\
\hline Satisfeita & 7 & $28 \%$ \\
\hline Pouco Satisfeita & - & - \\
\hline Insatisfeita & 3 & $12 \%$ \\
\hline
\end{tabular}

Tabela 5 - Índice de pacientes reoperadas.

\begin{tabular}{c|c|c}
\hline Complicações & Número de Pacientes & $\mathbf{\%}$ \\
\hline Nenhuma & 13 & $52 \%$ \\
\hline Correção da aréola & 10 & $40 \%$ \\
\hline Periareolar & 1 & $4 \%$ \\
\hline $\begin{array}{c}\text { Técnica de } \\
\text { Pitanguy }\end{array}$ & 1 & $4 \%$ \\
\hline
\end{tabular}

Tabela 8 - Somatória de um a dez das notas dadas.

\begin{tabular}{c|c|c|c|c|c|c|c|c|c|c}
\hline Notas & $\mathbf{1}$ & $\mathbf{2}$ & $\mathbf{3}$ & $\mathbf{4}$ & $\mathbf{5}$ & $\mathbf{6}$ & $\mathbf{7}$ & $\mathbf{8}$ & $\mathbf{9}$ & $\mathbf{1 0}$ \\
\hline Número de pacientes & 0 & 1 & 2 & 0 & 1 & 1 & 4 & 8 & 4 & 4 \\
\hline Porcentagem & - & $4 \%$ & $8 \%$ & - & $4 \%$ & $4 \%$ & $16 \%$ & $32 \%$ & $16 \%$ & $16 \%$ \\
\hline
\end{tabular}

\section{DISCUSSÃO}

Os procedimentos cirúrgicos foram baseados na técnica de Bustos $^{5}$, com algumas modificações que permitiram dispensar a inclusão de tela. A realização de ampla dissecção e ressecção do volume mamário quando necessário no pólo superior, seguida de deslizamento cranial dos segmentos medial e lateral do tecido glândulo-adiposo, a sutura entre si e na aponeurose do músculo peitoral maior permitem a hipercorreção do cone mamário. Esta manobra determina maior resistência à pressão contrária da sutura em "round-block”, que age ao longo dos anos, no sentido de alargar a cicatriz.

Não utilizamos tela nesta técnica, pois, apesar de seu emprego potencialmente propiciar maior benefício na qualidade do resultado, pode também apresentar maior 
índice de complicações. Os procedimentos que utilizam a técnica periareolar, especialmente sem o uso de tela, exigem menor ressecção de pele do que as técnicas com cicatrizes finais em $\mathrm{T}, \mathrm{L}$ ou mesmo as verticais. Esta peculiaridade limita a indicação deste procedimento para mamas pequenas e médias e sem grande flacidez cutânea, o que é mais comum entre pacientes mais jovens.

De acordo com os resultados obtidos, observamos uma análise muito positiva pelas pacientes com relação ao volume e à simetria final das mamas. Acreditamos que este aspecto está relacionado à evolução técnica, pela maior rotação dos retalhos laterais e mediais de tecido glândulo-adiposo, permitindo maior projeção do cone mamário, evitando as formas achatadas que eram observadas no início da utilização desta técnica. Com relação à forma e à ptose, as pacientes não ficaram tão satisfeitas como no tópico anterior. A ressecção limitada de pele, assim como a falta da elasticidade foram os fatores preponderantes neste quesito. Quanto ao aspecto das cicatrizes e à forma do CAP, foram encontradas as piores notas. Isto ocorre porque a tensão sofrida pela aréola na técnica de "round block" é maior do que em outras técnicas, resultando, algumas vezes, no alargamento das cicatrizes.

Quanto ao índice de satisfação, $88 \%$ das pacientes referiram estar muito satisfeitas ou satisfeitas, similar às avaliações nos outros tipos de técnicas ${ }^{13,14}$, comprovado pela consistência dos resultados. Dentre três pacientes não satisfeitas com resultado, uma foi reoperada pela mesma técnica e outra pela técnica de Pitanguy ${ }^{13}$; a terceira paciente não quis ser reoperada e não se submeteu a nenhum outro procedimento cirúrgico nas mamas.

A relevância deste trabalho é demonstrar uma avaliação tardia das pacientes. Sem dúvida, a técnica periareolar constitui outra opção para mamaplastias, com boa aceitação. A utilização do acesso periareolar tem aumentado, e como citado por Rohrich et al. ${ }^{13}$, "a técnica periareolar necessita maior revisão, pela menor satisfação por parte do médico, apesar de sua aplicação a um grande número de mamaplastias anualmente”.

A mamaplastia redutora periareolar é uma ótima opção em pacientes que necessitam de redução menor que $500 \mathrm{~g}$ em cada mama ${ }^{15}$, havendo autores dentre eles ${ }^{16,17}$ que utilizam a cirurgia periareolar com compensação vertical. Reiterando, temos realizado maior ressecção no pólo superior e maior deslizamento dos retalhos glandulares em bloco, com a aréola no sentido do pólo superior. A finalidade desta manobra é obter a hiperprojeção do cone mamário, de forma a resistir à pressão contrária da pele após a sutura em "round-block".

\section{CONCLUSÕES}

Os estudos estatísticos observados nas mamaplastias periareolares permite concluir ser um procedimento de elevada aceitação por parte das pacientes, pelos resultados a longo prazo e pela manutenção da qualidade estética, em função da existência de uma única cicatriz ao redor das aréolas. Como principais aspectos limitantes estão a indicação restrita a pacientes com pequenas e médias hipertrofias, com bom turgor de pele, e a tendência a alargamento da cicatriz.

\section{REFERÊNCIAS}

1. Rosel E, Starcks RB. Circum areolar mastopexy. Personal communication at the American Society of Plastic and Reconstructive Surgeons; 1973.

2. Andrews JM, Yshizuri MM, Martins DM, Ramos RR. An areolar approach to reduction mammaplasty. Br J Plast Surg. 1975;28(3):166-70.

3. Bartels RJ, Strickland DM, Douglas WM. A new mastopexy for mild or moderate breast ptosis. Plast Reconstr Surg. 1976;57(6):687-91.

4. Erol OO, Spira M. A mastopexy technique for mild to moderate ptosis. Plast Reconstr Surg. 1980;65(5):603-9.

5. Bustos RA. Periareolar mammaplasty with silicone supporting lamina. Plast Reconstr Surg. 1992;89(4):646-57

6. Góes JCS. Periareolar mammaplasty: double skin technique. Rev Soc Bras Cir Plast. 1989;4:55-63.

7. Góes JC. Periareolar mammaplasty: double skin technique with application of polyglactine or mixed mesh. Plast Reconstr Surg. 1996;97(5):5968.

8. Góes JC. Periareolar mastopexy: double skin technique with mesh support. Aesthet Surg J. 2003;23(2):129-35.

9. Benito J, Sanza IF. Periareolar techniques for mammary reduction and elevation. Aesthetic Plast Surg. 1993;17(4):311-6.

10. Aboudib JH Jr, Castro CC. Mammaplasty utilizing the periareolar approach. Aesthetic Plast Surg. 1998;22(1):51-7.

11. Felício Y. Periareolar reduction mammaplasty. Plast Reconstr Surg. 1991;88(5):789-98.

12. Fayman MS, Potgieter E, Becker PJ. Outcome study: periareolar mammaplasty patient's perspective. Plast Reconst Surg. 2003;111(2):676-84.

13. Rohrich RJ, Gosmann AA, Brown SA, Reisch J. Mastopexy references: a survey of board-certified plastic surgeons. Plast Reconst Surg. 2006;118(7):1631-8.

14. Cho BC, Yang JD, Baik BS. Periareolar reduction mammaplasty using an inferior dermal pedicle or a central pedicle. J Plast Reconstr Aesthet Surg. 2008;61(3):275-81.

15. Courtiss EH, Goldwyn RM. Reduction mammaplasty by the inferior pedicle technique. An alternative to free nipple and areola grafting for severe macromastia or extreme ptosis. Plast Reconstr Surg. 1977;59(4):500-7.

16. Martins PDE. Periareolar mammaplasty with flap transposition. Rev Bras Cir Plast. 1991;6(1-2):1-6.

17. Hammond DC, Alfonso D, Khuthaila DK. Mastopexy using the short scar periareolar inferior pedicle reduction technique. Plast Reconstr Surg. 2008;121(5):1533-9.
Correspondência para:
Ana Claudia Weck Roxo

Rua Ipanema, 21- Barra - Rio de Janeiro, RJ, Brasil - CEP 22631-390

E-mail: anacwroxo@rinet.com.br 